\title{
Determination of Spinal Anesthesia Induced Hypotension in Cesarean Section
}

\author{
Sanjay Kumar Gupta', Saurabh Singhal' \\ ${ }^{1}$ Assistant Professor, Department of Anaesthesia, FHMC, Tundla.
}

\section{Abstract}

Background: Spinal anesthesia is the global standard method of anesthesia for cesarean section. The present study was conducted to determine spinal anesthesia induced hypotension in cesarean section in study group. Subjects and Methods: The present study was conducted in the department of Anesthesia. It comprised of 68 pregnant women in age range 18-30 years. In all patients, ASA grade, gravidity, history of previous cesarean section and hypotension was recorded. Results: Age group 18-22 years had 32 patients, 23-27 years had 22 and 27-30 years had 14 patients. The difference was significant $(\mathrm{P}<0.05)$. ASA grade I was seen in 48 patients and II in 20 patients. Gravidity 1 was seen in 24 , 2 in 16, 3 in 15 and 4 in 13. 46 had 1 previous cesarean section and 22 had 2. The difference was significant $(\mathrm{P}<0.05)$. Mild hypotension was present in 2, moderate in 3 and severe in 6 . The difference was significant $(\mathrm{P}<0.05)$. Conclusion: Authors found that hypotension is frequently seen in cesarean section. In this study, 11 patients exhibited spinal anesthesia induced hypotension.

Keywords: Hypotension, Spinal anesthesia, Pregnant.

Corresponding Author: Dr. Saurabh Singhal, Assistant Professor, Department of Anaesthesia, FHMC, Tundla.

Received: July 2019

Accepted: July 2019

\section{Introduction}

Spinal anesthesia (SA) is the global standard method of anesthesia for cesarean section. However, SA induces maternal hypotension caused by aortocaval compression from the gravid uterus and sympathetic blockade which results in decreased arteriolar tone. Spinal anaesthesia can be associated with a number of problems, including hypotension and its sequelae. ${ }^{[1]}$ Hypotension causes problems for the mother and fetus, including nausea, vomiting, dizziness and fetal acidemia. Therefore, prediction and prevention of maternal hypotension are potentially important. Previous reports have assessed the prediction of maternal hypotension during cesarean section under SA; methods have included heart rate variability (HRV), pleth variability, index (PVI) and cerebral oxygen saturation $(\mathrm{ScO} 2)$. Furthermore, maternal hypotension may be predicted by a postural changeinduced increase in heart rate (HR) or comparison with preanesthetic HR. ${ }^{[2]}$

Regional or general anaesthesia (GA) are both acceptable for caesarean delivery, use of GA has decreased dramatically in the past few decades due to a higher risk of anaesthesia related maternal mortality. As a consequence, spinal anaesthesia (SA) is now the technique of choice for CS. Although SA is generally well tolerated, it is still associated with considerable side effects, the most common of which is maternal hypotension, potentially endangering both mother and child. ${ }^{[3]}$

The year 2002 marked the centenary of the first successful use of spinal anaesthesia for caesarean section by Hopkins who used it for a patient with placenta praevia. It is noteworthy that the technique was almost abandoned in its infancy because of problems associated with severe hypotension, which were exacerbated by the availability of only rudimentary monitoring and lack of awareness of the effects of vasodilatation and aortocaval compression. ${ }^{[4]}$ The present study was conducted to determine spinal anesthesia induced hypotension in cesarean section in study group.

\section{Subjects and Methods}

The present study was conducted in the department of Anesthesia. It comprised of 68 pregnant women in age range 18-30 years with American Society of Anaesthesiologists' (ASA) physical status grade I or II who were scheduled to undergo elective cesarean section.

Patient information such name, age etc. was recorded. In all patients, ASA grade, gravidity and history of previous cesarean section was recorded. Mild hypotension was defined as a drop of $\geq 10 \%$ and $\leq 20 \%$ in baseline SBP, moderate hypotension defined as a drop of $>20 \%$ and $\leq 30 \%$ in baseline SBP and severe hypotension defined as a drop of $>30 \%$ in baseline SBP. Results were tabulated and subjected to statistical analysis. P value less than 0.05 was considered significant. 
Results

\begin{tabular}{|l|l|l|}
\hline Table 1: Age wise distribution of patients \\
\hline Age group (Years) & Number & P value \\
\hline $18-22$ & 32 & 0.01 \\
\hline $23-27$ & 22 & \\
\hline $28-30$ & 14 & \\
\hline
\end{tabular}

[Table 1] shows that age group 18-22 years had 32 patients, 23-27 years had 22 and 27-30 years had 14 patients. The difference was significant $(\mathrm{P}<0.05)$.

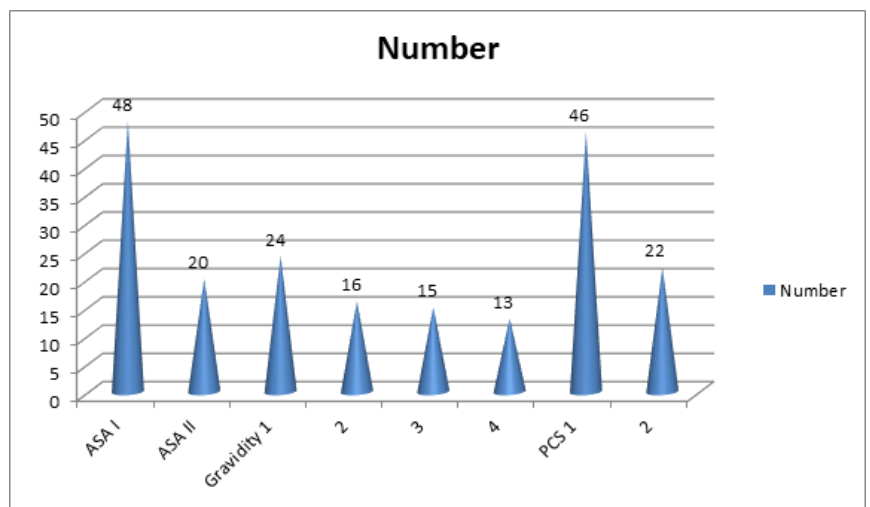

Figure 1: Assessment of parameters

[Figure 1] shows that ASA grade I was seen in 48 patients and II in 20 patients. Gravidity 1 was seen in 24,2 in 16,3 in 15 and 4 in 13. 46 had 1 previous cesarean section and 22 had 2. The difference was significant $(P<0.05)$.

\section{Table 2: Prevalence of hypotension}

\begin{tabular}{|l|l|l|}
\hline Hypotension & Number & P value \\
\hline Mild & 2 & 0.01 \\
\hline Moderate & 3 & \\
\hline Severe & 6 & \\
\hline
\end{tabular}

[Table 2\& Figure 1] shows that mild hypotension was present in 2, moderate in 3 and severe in 6 . The difference was significant $(\mathrm{P}<0.05)$.

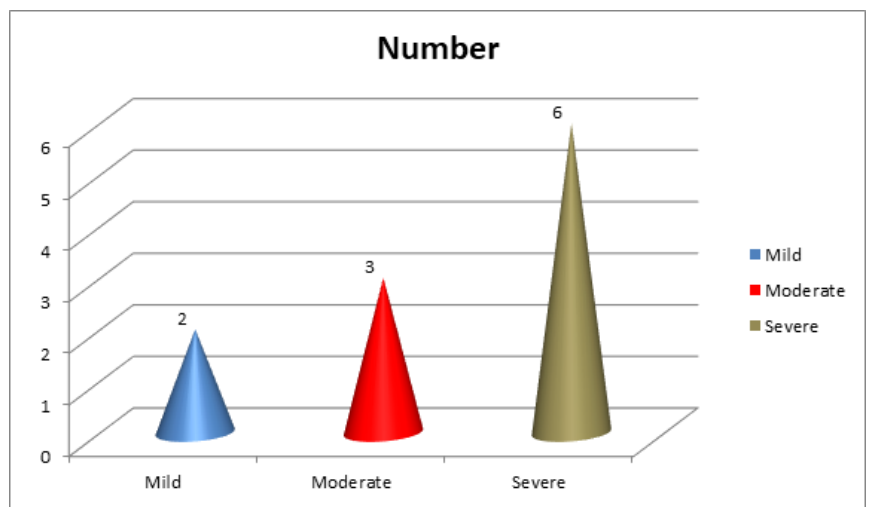

Figure 2: Prevalence of hypotension

\section{Discussion}

Spinal hypotension is common in women who receive spinal anaesthesia for Caesarean section, with an incidence of up to $71 \%$. Spinal hypotension can occur precipitously and, if severe, can result in important perinatal adverse outcomes, such as maternal nausea and vomiting, fetal acidosis and may be an important contributory factor for maternal death related to regional anaesthesia. Mothers with pre-delivery hypovolaemia may be at risk of cardiovascular collapse because the sympathetic blockade may severely decrease venous return. As a consequence, prevention of spinal hypotension has been a key research area within the field of obstetric anaesthesia. ${ }^{[5]}$ The present study was conducted to determine spinal anesthesia induced hypotension in cesarean section in study group.

In present study, age group 18-22 years had 32 patients, 2327 years had 22 and 27-30 years had 14 patients. We found that ASA grade I was seen in 48 patients and II in 20 patients. Gravidity 1 was seen in 24,2 in 16,3 in 15 and 4 in 13. 46 had 1 previous cesarean section and 22 had 2. Mitra et $\mathrm{al}{ }^{[6]}$ revealed that the incidence of mild, moderate and severe hypotension was 20\%, 35\% and 40\%, respectively. Eventually, ten risk factors were found to be associated with hypotension, including age $>35$ years, body mass index $\geq 25$ $\mathrm{kg} / \mathrm{m} 2,11-20 \mathrm{~kg}$ weight gain, gravidity $\geq 4$, history of hypotension, baseline systolic blood pressure (SBP) $<120$ $\mathrm{mmHg}$ and baseline heart rate $>100$ beats $/ \mathrm{min}$ in maternal modeling, fluid preloading $\geq 1000 \mathrm{ml}$, adding sufentanil to bupivacaine and sensory block height >T4 in anaesthesia related modeling.

We found that hypotension was observed in 11 patients. We found that mild hypotension was present in 2 , moderate in 3 and severe in. ${ }^{[6]}$ Spinal anaesthesia is produced by the injection of local anaesthetic, often together with an opioid adjunct, into the subarachnoid space, with the objective of blocking conduction in afferent sensory fibres that transmit pain impulses to the brain. There is also an increase in sympathetic versus parasympathetic activity which predisposes to a greater degree of peripheral vasodilation. ${ }^{[7]}$ Epidural blockade produces a similar extent of sympathectomy, but there is a lower incidence and severity of hypotension since the rate of onset of sympathectomy is slower, allowing more time for cardiovascular compensation. ${ }^{[8]}$

The main cause of maternal hypotension during SA for cesarean section is peripheral vasodilation, and it has been reported that the degree of decrease of $\mathrm{BP}$ and systemic vascularresistance depend on the dose of local anesthetic. Identification of patients at risk of hypotension is important for anesthesia management and maternal and fetal safety. ${ }^{\left[{ }^{[9}\right.}$ Prophylaxis of maternal hypotension has been investigated in numerous studies. Various fluid protocols have reduced but not prevented of hypotension, hence the importance of early administration of vasopressors.

Some predictive factors of maternal hypotension during cesarean section under SA have been reported previously: increase in HR by $>10$ beats/min, leg flexion or complaint of dysphoria following postural change from left lateral to supine position, high PVI before anesthesia, decrease of $\mathrm{ScO} 2$ under $\mathrm{SA}$ and pre-anesthesia $\mathrm{HR}<71$ or $>89$ beats/min. ${ }^{[10,11]}$

Spinal anaesthesia is produced by the injection of local anaesthetic, often together with an opioid adjunct, into the subarachnoid space, with the objective of blocking conduction in afferent sensory fibres that transmit pain 
impulses to the brain. ${ }^{[12]}$ However, conduction block from local anaestheticsis non-specific and preganglionic fibres to the sympathetic chain are also affected, resulting in sympathetic block and hypotension which can cause hypoperfusion of the uterus and placenta. ${ }^{[13]}$

In pregnant women, greater sensitivity to local anaesthetics results in higher blocks, and compounded by the effects of aortocaval compression, hypotension occurs with greater frequency and severity. ${ }^{[14]}$ Chinachoti et al, ${ }^{[15]}$ suggested that Previously, maternal hypotension and fetal outcome were thought to be improved by avoiding aortocaval compression (left uterine displacement) and increasing the blood volume, such as by intravenous fluid loading to increase the venous return, cardiac filling pressure, and cardiac output (CO). These techniques, however, have proven ineffective, and use of vasopressors is the most reliable method for countering the hypotension induced by spinal anesthesia.

Vasopressor drugs act on $\alpha 1$-, $\beta 1$ - and $\beta 2$-adrenoreceptors in the heart and vascular system. The physiological response of these adrenoreceptor agonists depends on the type and location of the receptors. ${ }^{[16]}$ Vasoconstriction is mainly mediated by $\alpha 1$-receptors. However, some vasopressors can also stimulate $\beta 1$ - and/or $\beta 2$-receptors directly or indirectly, leading to positive inotropic (increasing cardiac contractility) and/or positive chronotropic (increasing heart rate, HR) effects. The complex hemodynamic effects of the various vasoconstrictors depend on the relative stimulation of these adrenoreceptors. ${ }^{[17]}$ Reflex cardiovascular responses to vasopressors, on the other hand, may result in other changes, including the unwanted reflex bradycardia.

The limitation of the study is small sample size. Inclusion of large sample could show different results. Large scale studis are required to to substantiate the results.

\section{Conclusion}

Authors found that hypotension is frequently seen in cesarean section. In this study, 11 patients exhibited spinal anesthesia induced hypotension.

\section{References}

1. Brenck F, Hartmann B, Katzer C, Obaid R, Brüggmann D, Benson M, et al. Hypotension after spinal anaesthesia for cesarean section: Identification of risk factors using an anaesthesia information management system. J ClinMonitComput 2009;23:85-92.

2. Ohpasanon P, Chinachoti T, Sriswasdi P, Srichu S. Prospective study of hypotension after spinal anaesthesia for cesarean section at Siriraj Hospital: Incidence and risk factors, part 2. J Med Assoc Thai 2008;91:675-80.

3. Reynolds F, Seed PT. Anaesthesia for caesarean section and neonatal acid-base status: A meta-analysis. Anaesthesia 2005;60:636-53.

4. Carpenter RL, Caplan RA, Brown DL, Stephenson C, Wu R. Incidence and risk factors for side effects of spinal anaesthesia. Anaesthesiology 1992;76:906-16.

5. Jadon A. Complications of regional and general anaesthesia in obstetric practice. Indian J Anaesth 2010;54:415-20.

6. Mitra JK, Roy J, Bhattacharyya P, Yunus M, Lyngdoh NM. Changing trends in the management of hypotension following spinal anaesthesia in cesarean section. J Postgrad Med2013;59:121-6.

7. Maayan-Metzger A, Schushan-Eisen I, Todris L, EtchinA, Kuint J. Maternal hypotension during elective cesarean section and short-term neonatal outcome. Am J ObstetGynecol 2010;202:1-5.

8. Bishop DG. Predicting spinal hypotension during Cesarean section. South Afr J AnaesthAnalg 2014;20:170-3.

9. Hawkins JL, Koonin LM, Palmer SK, Gibbs CP. Anaesthesia-related deaths during obstetric delivery in the United States, 1979-1990. Anaesthesiology 1997;86:277-84.

10. Orbach-Zinger S, Ginosar Y, Elliston J, Fadon C, Abu-Lil M, Raz A, et al. Influence of preoperative anxiety on hypotension after spinal anaesthesia in women undergoing caesareandelivery. $\mathrm{Br} \mathrm{J}$ Anaesth 2012;109:943-9.

11. Rout CC, Rocke DA. Prevention of hypotension following spinal anaesthesia for caesarean section. IntAnaesthesiolClin 1994;32:117-36.

12. Mercier FJ, Augè M, Hoffmann C, Fischer C, Le Gouez A. Maternal hypotension during spinal anaesthesia for caesarean delivery. Minerva Anestesiol 2013;79:62-73.

13. Palmese S, Manzi M, Visciano V, Scibilia A, Natale A. Reduced hypotension after subarachnoid anaesthesia with ondansetron most colloids in parturients undergoing caesarean section. A retrospective study. Internet J Anaesthesiol 2012;30:1178-85.

14. Hu J, Zhang C, Yan J, Wang R, Wang Y, Xu M, et al. Sufentanil and bupivacaine combination versus bupivacaine alone for spinal anaesthesia during cesarean delivery: A Meta-analysis of randomized trials. PLoS One 2016;11:0152605.

15. Chinachoti $\mathrm{T}$, Saetia $\mathrm{S}$, Chaisiri $\mathrm{P}$, Tritrakarn $\mathrm{T}$. Incidence and risk factors of hypotension and bradycardia during spinal anaesthesia. Siriraj Med J 2006;58:696-701.

16. Olofsson C, Nygårds EB, Bjersten AB, Hessling A. Low-dose bupivacaine with sufentanil prevents hypotension after spinal anaesthesia for hip repair in elderly patients. ActaAnaesthesiolScand 2004;48:1240-4.

17. Frölich MA, Caton D. Baseline heart rate may predict hypotension after spinal anaesthesia in prehydrated obstetrical patients. Can J Anaesth 2002;49:185-9.

Copyright: (c) the author(s), publisher. Academia Anesthesiologica Internationalis an Official Publication of "Society for Health Care \& Research Development". It is an open-access article distributed under the terms of the Creative Commons Attribution Non-Commercial License, which permits unrestricted non-commercial use, distribution, and reproduction in any medium, provided the original work is properly cited.

How to cite this article: Gupta SK, Singhal S.Determination of Spinal Anesthesia Induced Hypotension in Cesarean Section. Acad. Anesthesiol. Int. 2019;4(2):71-73.

DOI: dx.doi.org/10.21276/aan.2019.4.2.19

Source of Support: Nil, Conflict of Interest: None declared. 